RESCEU-02/11

\title{
$f(R)$ Gravity and its Cosmological Implications
}

\author{
Hayato Motohashi ${ }^{1,2}$, Alexei A. Starobinsky ${ }^{2,3}$, and Jun'ichi Yokoyama ${ }^{2,4}$ \\ 1 Department of Physics, Graduate School of Science, \\ The University of Tokyo, Tokyo 113-0033, Japan \\ ${ }^{2}$ Research Center for the Early Universe (RESCEU), \\ Graduate School of Science, The University of Tokyo, Tokyo 113-0033, Japan \\ ${ }^{3}$ L. D. Landau Institute for Theoretical Physics, Moscow 119334, Russia \\ ${ }^{4}$ Institute for the Physics and Mathematics of the Universe(IPMU), \\ The University of Tokyo, Kashiwa, Chiba, 277-8568, Japan
}

\begin{abstract}
We have investigated the evolution of a homogeneous isotropic background of the Universe and inhomogeneous subhorizon matter density perturbations in viable $f(R)$ models of present dark energy and cosmic acceleration analytically and numerically. It is found that viable $f(R)$ models generically exhibit recent crossing of the phantom boundary $w_{\mathrm{DE}}=-1$. Furthermore, it is shown that the growth index of perturbations depends both on time and wavenumber. This anomalous growth may explain properties of the observational matter power spectrum from the SDSS data and can also partially counteract the spectrum suppression by massive neutrinos making larger values of the total sum of neutrino rest masses possible.
\end{abstract}




\section{INTRODUCTION}

Although the standard spatially flat $\Lambda$-Cold-Dark-Matter $(\Lambda \mathrm{CDM})$ model can explain cosmic acceleration and is consistent with current observational data[1], the observed value of the cosmological constant term is much smaller than any other energy scale known in physics. On the other hand, we are sure that "primordial dark energy (DE)", which is responsible for inflation in the early universe [2-4], is not identical to the cosmological constant, in particular, it is not stable and eternal. Hence, it is natural to seek nonstationary models of the current DE, too.

$f(R)$ gravity is one of those dynamical DE models in which the Hilbert-Einstein action is modified and generalized by incorporating a new phenomenological function of the Ricci scalar $R, f(R)$. This theory provides a self-consistent and nontrivial alternative to the $\Lambda$ CDM model. It contains a new scalar degree of freedom dubbed "scalaron" in Ref. [2]. The existence of this additional degree of freedom imposes a number of conditions on viable functional forms of $f(R)$ for $R \gg R_{0}$ and up to curvatures $R$ in the center of neutron stars:

$$
|f(R)-R| \ll R, \quad\left|f^{\prime}(R)-1\right| \ll 1, \quad R f^{\prime \prime}(R) \ll 1
$$

where the prime denotes the derivative with respect to the argument $R$ and $R_{0}$ is a parameter

of the order of the present Ricci curvature. Furthermore, $f(R)$ should satisfy the stability conditions:

$$
f^{\prime \prime}(R)>0, \quad f^{\prime}(R)>0 .
$$

Specific functional forms satisfying all these conditions have been proposed in Refs. [5-7].

In this paper, we have carried out numerical calculations of the evolution of both a background space-time and density fluctuations for the particular $f(R)$ model introduced in Ref. [7]. As a result, we have found the phantom boundary crossing at an intermediate redshift $z \lesssim 1$ for the background space-time metric and an anomalous behaviour of the growth of density fluctuations. 

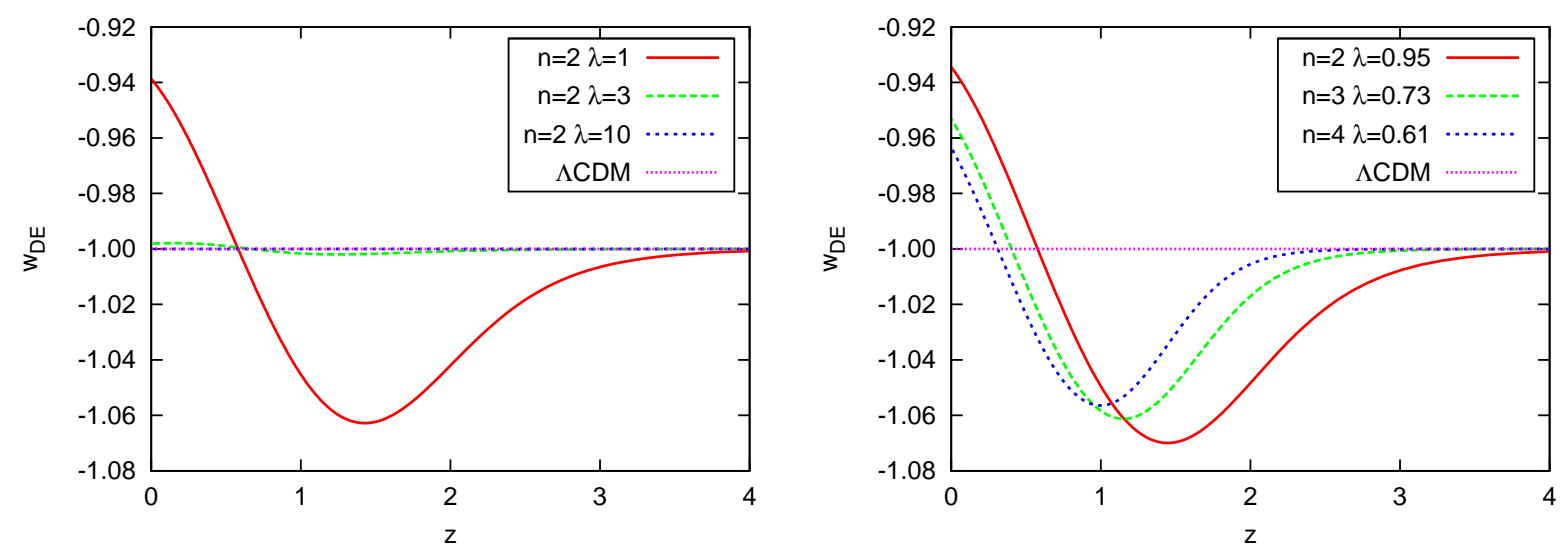

FIG. 1: Evolution of the equation-of-state parameter $w_{\mathrm{DE}}(z)$ for the effective dark energy.

\section{BACKGROUND UNIVERSE}

We adopt the following action with a three-parameter family of $f(R)$ models:

$$
\begin{aligned}
& S=\frac{1}{16 \pi G} \int d^{4} x \sqrt{-g} f(R)+S_{m}, \\
& f(R)=R+\lambda R_{s}\left[\left(1+\frac{R^{2}}{R_{s}^{2}}\right)^{-n}-1\right],
\end{aligned}
$$

where $n, \lambda$ and $R_{s}$ are model parameters and $S_{m}$ is the action of the matter content. We can derive field equations from the action (3) and rewrite them as

$$
\begin{aligned}
R_{\nu}^{\mu}-\frac{1}{2} \delta_{\nu}^{\mu} R & =-8 \pi G\left(T_{\nu(m)}^{\mu}+T_{\nu(\mathrm{DE})}^{\mu}\right), \\
8 \pi G T_{\nu(\mathrm{DE})}^{\mu} & \equiv(F-1) R_{\nu}^{\mu}-\frac{1}{2}(f-R) \delta_{\nu}^{\mu}+\left(\nabla^{\mu} \nabla_{\nu}-\delta_{\nu}^{\mu} \square\right) F,
\end{aligned}
$$

where $F(R) \equiv f^{\prime}(R)$. Working in the spatially flat Friedmann-Robertson-Walker (FRW) space-time with the scale factor $a(t)$, we find

$$
\begin{aligned}
3 H^{2} & =8 \pi G \rho-3(F-1) H^{2}+\frac{1}{2}(F R-f)-3 H \dot{F}, \\
2 \dot{H} & =-8 \pi G \rho-2(F-1) \dot{H}-\ddot{F}+H \dot{F},
\end{aligned}
$$

where $H \equiv \dot{a} / a$ is the Hubble parameter and $\rho$ is the energy density of matter.

From Eq. (6), we can express the effective energy density and pressure of dark energy as

$$
\begin{aligned}
8 \pi G \rho_{\mathrm{DE}} & =-3 H \dot{R} F^{\prime}+3\left(H^{2}+\dot{H}\right)(F-1)-\frac{1}{2}(f-R), \\
8 \pi G\left(\rho_{\mathrm{DE}}+P_{\mathrm{DE}}\right) & =2 \dot{H}(F-1)-H \dot{F}+\ddot{F}
\end{aligned}
$$


TABLE I: $B(0)$ for various model parameters.

\begin{tabular}{ll|c}
$n$ & $\lambda$ & $B(0)$ \\
\hline 2 & 0.95 & $2.09 \times 10^{-1}$ \\
2 & 4 & $9.36 \times 10^{-4}$ \\
2 & 8 & $6.07 \times 10^{-5}$ \\
3 & 0.73 & $1.86 \times 10^{-1}$ \\
3 & 2 & $1.34 \times 10^{-3}$ \\
3 & 3 & $1.35 \times 10^{-4}$ \\
4 & 0.61 & $1.73 \times 10^{-1}$ \\
4 & 1 & $1.33 \times 10^{-2}$
\end{tabular}

respectively where $R=12 H^{2}+6 \dot{H}$. We define the DE equation of state parameter $w_{\mathrm{DE}}$ by the ratio $w_{\mathrm{DE}} \equiv P_{\mathrm{DE}} / \rho_{\mathrm{DE}}$,

$$
w_{\mathrm{DE}} \equiv \frac{P_{\mathrm{DE}}}{\rho_{\mathrm{DE}}}=-1+\frac{2 \dot{H}(F-1)-H \dot{F}+\ddot{F}}{-3 H \dot{R} F^{\prime}+3\left(H^{2}+\dot{H}\right)(F-1)-(f-R) / 2} .
$$

For the appropriate initial condition corresponding to the existence of cosmic inflation in the past, $f-R$ acquires an asymptotically constant value $f-R=-\lambda R_{s}$ at high redshift. In this regime the evolution of the Universe is the same as that obtained from the Einstein action with a cosmological constant $\Lambda(\infty)=\lambda R_{s} / 2$.

The late-time asymptotic de Sitter solution has a curvature $R=R_{1}$ which is obtained as the maximal solution of the equation $2 f\left(R_{1}\right)=R_{1} f^{\prime}\left(R_{1}\right)$, namely,

$$
\alpha(r) \equiv r+2 \lambda\left[\frac{1+(n+1) r^{2}}{\left(1+r^{2}\right)^{n+1}}-1\right]=0,
$$

where $r \equiv R_{1} / R_{s}$. It is obvious that the Minkowski space-time, $r=0$, is one of the solutions. The stability condition of this future de Sitter solution $[8,16], f^{\prime}\left(R_{1}\right)>R_{1} f^{\prime \prime}\left(R_{1}\right)$, imposes the following constraint on $r$,

$$
\beta(r) \equiv \frac{\left(1+r^{2}\right)\left[\left(1+r^{2}\right)^{n+1}-2 n \lambda r\right]}{2 n \lambda\left[(2 n+1) r^{2}-1\right]}-r>0,
$$

which is stronger than any other constraint discussed above. For each $n$, we can find $r$, which marginally satisfies Eq. (13) and gives the minimal allowed value of $\lambda$. Numerically we find $\left(n, r_{\min }, \lambda_{\min }\right)=(2,1.267,0.9440),(3,1.041,0.7259)$, and $(4,0.9032,0.6081)$ for $n \leq 4$. 
We numerically solve the evolution equation (8) using Eq. (7) to check the numerical accuracy and taking $t_{i}$ as the moment of time when the matter density parameter was $\Omega_{i}=16 \pi G \rho_{i} /\left(16 \pi G \rho_{i}+\lambda R_{s}\right)=0.998$. We determine the current epoch by the requirement that the value of $\Omega$ takes the observed central value $\Omega_{0}=0.27$. Then $R_{s}$ is fixed in such a way that the current Hubble parameter $H_{0}=72 \mathrm{~km} / \mathrm{s} / \mathrm{Mpc}$ is reproduced.

Figure 1 depicts the evolution of $w_{\mathrm{DE}}$ as a function of redshift $z$ [14]. Phantom crossing is manifest there. As expected, $w_{\mathrm{DE}}$ approaches $-1=$ constant as we increase $\lambda$ for fixed $n$. For $\lambda=\lambda_{\min }$, deviations from $w_{\mathrm{DE}}=-1$ are observed at $\sim 5 \%$ level in both directions for $z \lesssim 2$ independently of $n$. Such behaviour of $w_{\mathrm{DE}}$ is well admitted by all the most recent observational data[1]. The average value of $w_{\mathrm{DE}}$ over the interval $0 \leq z \leq 1$ to which all $\mathrm{BAO}$ and most of the $\mathrm{SN}$ data refer is very close to -1 . Moreover, in this range the behaviour of $w_{\mathrm{DE}}$ for $\lambda=\lambda_{\min }$ is well fitted by the CPL fit [9] $w_{\mathrm{DE}}(z)=w_{0}+w_{a} z /(1+z)$ with $\left(n, w_{0}, w_{a}\right)=(2,-0.92,-0.23),(3,-0.94,-0.22)$, and $(4,-0.96,-0.21)$, respectively. These values of $w_{0}$ and $w_{a}$ lie very close to the center of the $68 \%$ and $95 \%$ CL ellipses for all combined data in Fig. 13 of Ref. [1]. This phantom crossing is not peculiar to the specific choice of the function (4) but a generic one for models which satisfy the stability condition $F^{\prime}>0$.

We have also calculated the quantity $B(z)=\left(f^{\prime \prime} / f^{\prime}\right)(d R / d \ln H)$ at the present time. The results are presented in Table I.

\section{DENSITY FLUCTUATIONS}

Armed with the evolution of the Universe background, we proceed to the investigation of the evolution of matter density fluctuations, $\delta$, in $f(R)$ gravity. In the subhorizon limit, the evolution equation is derived as $[10,11]$

$$
\begin{aligned}
& \ddot{\delta}+2 H \dot{\delta}-4 \pi G_{\mathrm{eff}} \rho \delta=0, \\
& G_{\mathrm{eff}}=\frac{G}{F} \frac{1+4 \frac{k^{2}}{a^{2}} \frac{F^{\prime}}{F}}{1+3 \frac{k^{2}}{a^{2}} \frac{F^{\prime}}{F}} .
\end{aligned}
$$

Equation (14) reduces to the correct evolution equation for all wavenumbers for the $\Lambda \mathrm{CDM}$ model in the Einstein gravity where $F=1$. The time and $k$-dependence of the effective gravitational constant $G_{\text {eff }}$ changes the evolution of density fluctuations. 

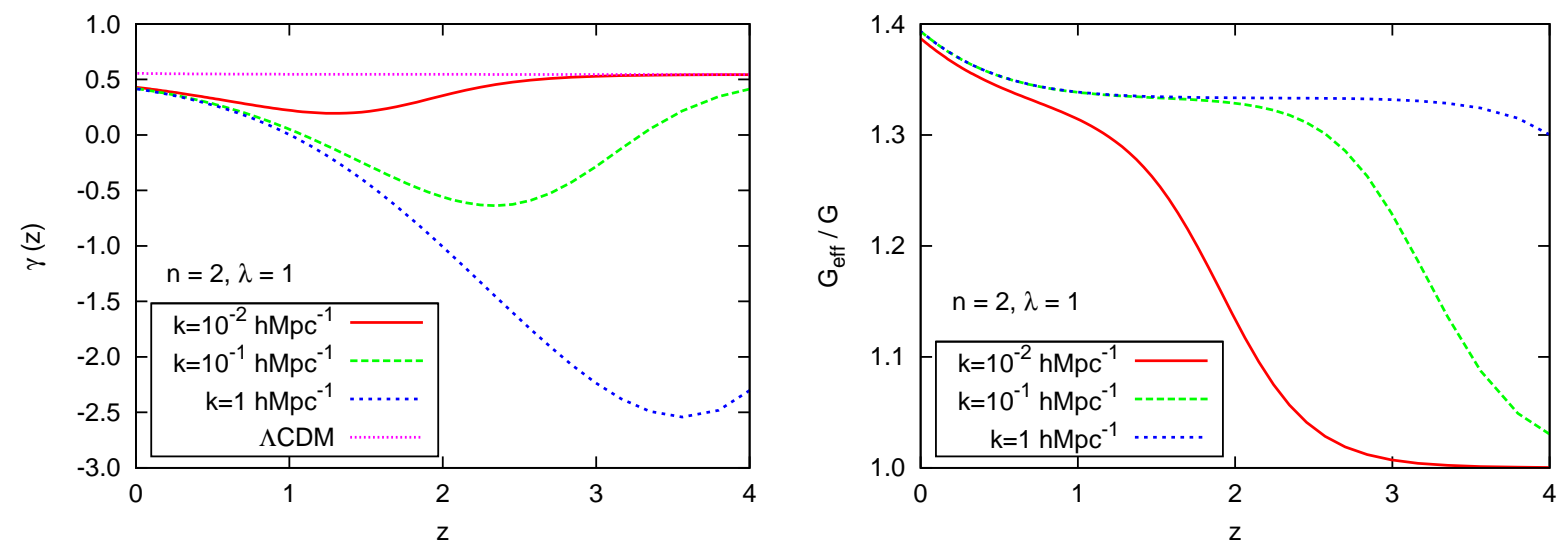

FIG. 2: Evolutions of the growth index $\gamma(z)$ and the effective gravitational constant $G_{\text {eff }}(z)$ for $n=2$ and $\lambda=1$.

First, let us consider the gravitational growth index, $\gamma(z)$, which is an important quantity helping to distinguish different modified theories of gravity. It is defined through

$$
\frac{d \ln \delta}{d \ln a}=\Omega_{m}(z)^{\gamma(z)}, \quad \text { or } \quad \gamma(z)=\frac{\log \left(\frac{\dot{\delta}}{H \delta}\right)}{\log \Omega_{m}}
$$

In the standard $\Lambda \mathrm{CDM}$ model, it takes a practically constant value $\gamma \cong 0.55$. However, it evolves with time in modified gravity theories in general. We also note that $\gamma(z)$ has a nontrivial $k$-dependence in $f(R)$ gravity, since density fluctuations with different wavenumbers evolve differently. Therefore, this quantity is a useful diagnostic to distinguish DE in modified gravity from the $\Lambda \mathrm{CDM}$ model in the Einstein gravity. We present the evolution of $\gamma(z)$ together with that of $G_{\text {eff }} / G$ for different values of $k$ in Fig. 2. $\gamma(z)$ takes a constant value identical to the $\Lambda \mathrm{CDM}$ model in the early high-redshift regime because $f(R)$ gravity is indistinguishable from the Einstein gravity plus a positive cosmological constant then. It gradually decreases with time, reaches a minimum, and then increases again towards the present epoch. Current constraints on the growth index[12] are not strong enough to detect any deviation from the $\Lambda$ CDM model and/or to obtain new bounds on $f(R)$ DE models, but future observations may reveal its time and wavenumber dependences.

Second, we focus on the additional late-time transfer function for linear matter perturbations arising in $f(R)$ gravity. The evolution equation (14) can be solved analytically in the high-curvature regime when the scale factor evolves as $a(t) \propto t^{2 / 3}$ and $F$ takes the asymptotic form $F \simeq 1-2 n \lambda\left(R / R_{s}\right)^{-2 n-1} \equiv 1-\left(R / R_{c}\right)^{-N-1}$, where $N=2 n$ and $R_{c}=R_{s}(2 n \lambda)^{1 /(2 n+1)}$. 

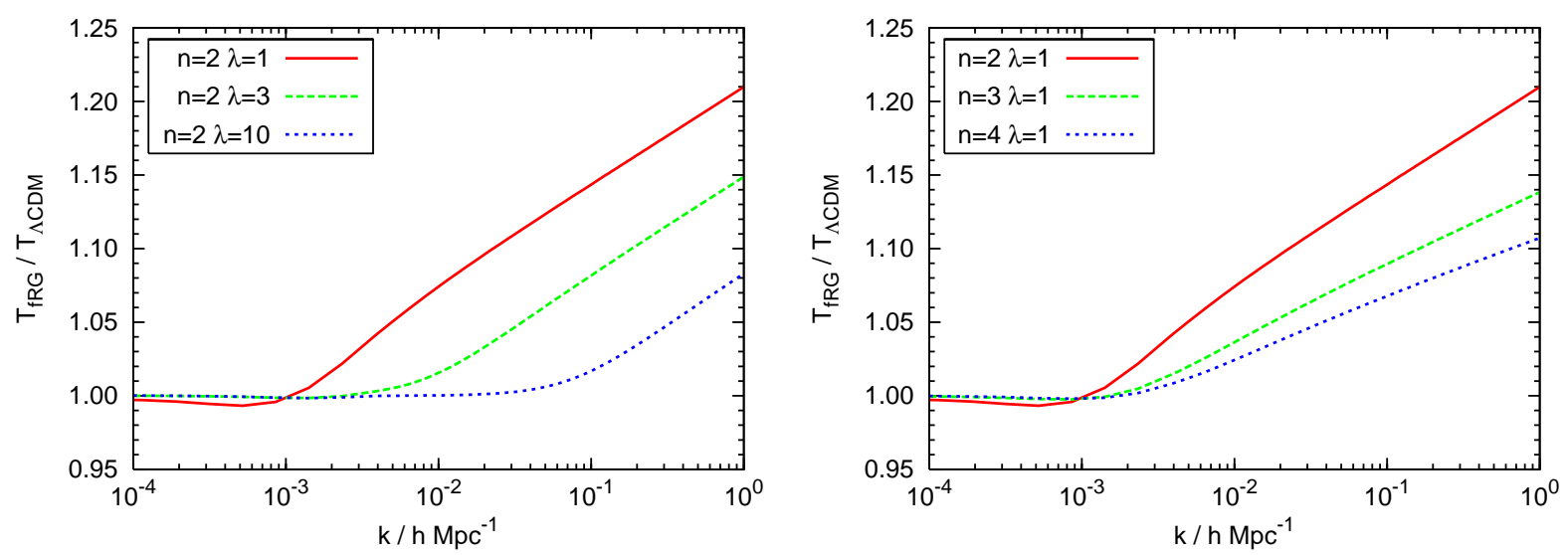

FIG. 3: Additional transfer function $C(k)$ in $f(R)$ gravity.

The two independent solutions of Eq. (14) in this regime read

$$
\begin{aligned}
& \delta_{k}(t)=\delta_{i k}\left(\frac{t}{t_{i}}\right)^{\frac{-1 \pm 5}{6}} \\
& \times{ }_{2} F_{1}\left(\frac{ \pm 5-\sqrt{33}}{4(3 N+4)}, \frac{ \pm 5+\sqrt{33}}{4(3 N+4)} ; 1 \pm \frac{5}{2(3 N+4)} ;-3 \frac{(N+1) k^{2}}{a_{i}^{2} R_{c}^{2}}\left(\frac{t}{t_{i}}\right)^{2 N+8 / 3}\right)
\end{aligned}
$$

in terms of the hypergeometric function[13]. Hereafter, we consider the upper sign solution only, because the other solution corresponds to the decaying mode and is singular at $t \rightarrow 0$. Then, the solution behaves as

$$
\delta_{k}(t) \stackrel{t \rightarrow 0}{\longrightarrow} \delta_{i k}\left(\frac{t}{t_{i}}\right)^{\frac{2}{3}} \text { and } \delta_{k}(t) \stackrel{t \rightarrow \infty}{\longrightarrow} \delta_{i k} C(k)\left(\frac{t}{t_{i}}\right)^{\frac{-1+\sqrt{33}}{6}},
$$

respectively. The transfer function, $C(k)$, is given by

$$
C(k)=\frac{\Gamma\left(1+\frac{5}{2(3 N+4)}\right) \Gamma\left(\frac{\sqrt{33}}{2(3 N+4)}\right)}{\Gamma\left(1+\frac{5+\sqrt{33}}{4(3 N+4)}\right) \Gamma\left(\frac{5+\sqrt{33}}{4(3 N+4)}\right)}\left[\frac{3(N+1) k^{2}}{a_{i}^{2} R_{c}}\left(\frac{3 R_{c} t_{i}^{2}}{4}\right)^{N+2}\right]^{\frac{-5+\sqrt{33}}{4(3 N+4)}},
$$

where $t_{i}=2 / 3 \sqrt{6 / \lambda R_{s}} \sinh ^{-1} \sqrt{\left(1-\Omega_{i}\right) / \Omega_{i}}$. We have confirmed the additional transfer function $C(k)$ numerically as Fig. 3 .

Finally, we proceed to compare the results of our numerical calculations for matter power spectrum with observational data from SDSS DR7[17]. The left panel of Fig. 4 shows the fitting of the matter power spectrum for the $\Lambda$ CDM model. The vertical axis is the ratio of the power spectrum normalized by the power spectrum in $\Lambda$ CDM model with bias parameter $b=1.25$. The data with error bars are LRG samples in SDSS DR7. As far as we use a 

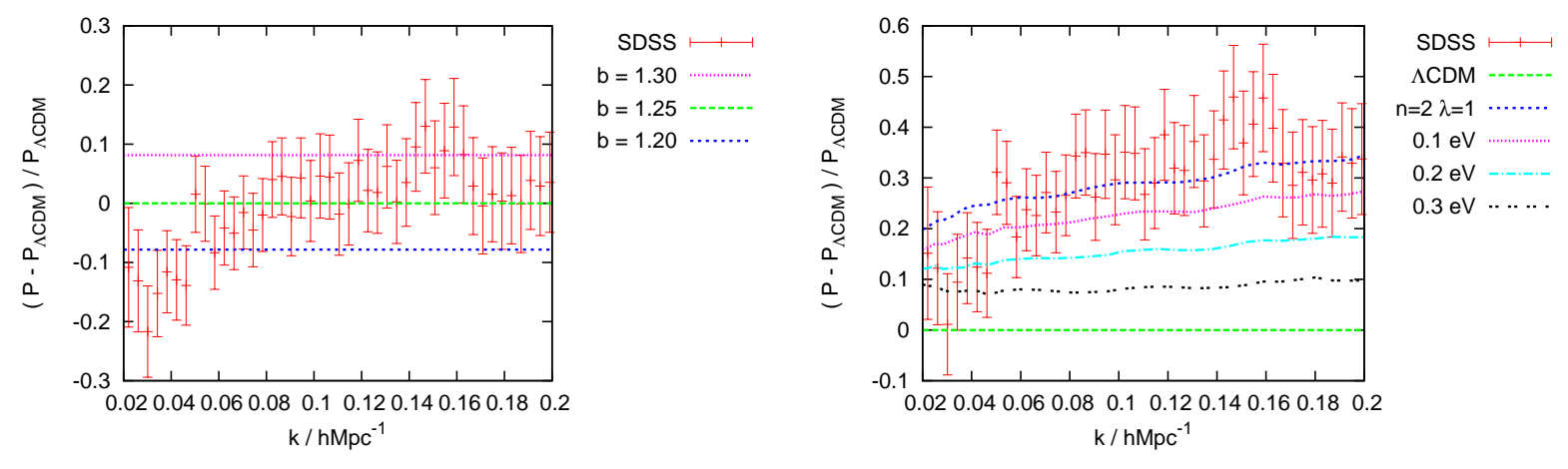

FIG. 4: Power spectrum with SDSS data. Left: Power spectrum in the $\Lambda$ CDM model normalized by $P_{\Lambda \mathrm{CDM}}(b=1.25)$. Right: Power spectrum in $f(R)$ gravity for $n=2$ and $\lambda=1$ with total neutrino mass up to $0.3 \mathrm{eV}$ normalized by $P_{\Lambda \mathrm{CDM}}(b=1.1)$.

constant bias, we see that observational data are increasing with wavenumber compared with the theoretical expectation line in the $\Lambda \mathrm{CDM}$ model. Furthermore, if we include massive neutrinos, the fitting becomes worse because their free streaming suppress the matter power spectrum. We have found that $f(R)$ gravity enhances the matter power spectrum and produces a better fit to the SDSS data than the $\Lambda$ CDM model without introducing a scale dependent bias or a nonlinear bias, see the right panel of Fig. 4. There is also a possibility to admit a larger total sum of neutrino restmasses compared to the $\Lambda$ CDM model since the anomalous growth of perturbations partially counteracts their suppression by free streaming of massive neutrinos [15].

\section{CONCLUSION}

In this paper, we have numerically calculated the evolution of both a homogeneous isotropic background and matter density fluctuations in a viable $f(R)$ DE model based on the specific functional form proposed in Ref. [7]. We have found that viable $f(R)$ gravity models of the present DE and accelerated expansion of the Universe generically exhibit phantom behaviour during the matter-dominated stage with crossing of the phantom boundary $w_{\mathrm{DE}}=-1$ at redshifts $z \lesssim 1$. More exactly, this behaviour is characteristic for all $f(R) \mathrm{DE}$ models that have $f^{\prime \prime}(R)>0$ and a stable future de Sitter epoch and which approach the Einstein gravity sufficiently fast for $R \gg R_{0}$, under the condition that the gravitational constant $G$ in the Einsteinian representation of the field equations (4) is normalized to its value 
measured in laboratory experiments (i.e., for $R \gg R_{0}$, too). The predicted time evolution of $w_{\mathrm{DE}}$ has qualitatively the same behaviour as that has recently obtained from observational data[18]. However, it is important that the condition of stability, or even metastability, of the future de Sitter epoch strongly restricts the possible deviation of $w_{\mathrm{DE}}$ from -1 by several percents in these models. Thus, the DE phantomness should be small, if it exists at all, that agrees well with the present observational data.

As for the density fluctuations, we have also investigated the growth index $\gamma(k, z)$ of density fluctuations and have presented an explanation of its anomalous evolution in terms of the time dependence of $G_{\text {eff }}$. Note that this evolution is characteristic for all $f(R)$ models in which the scalar particle (scalaron) becomes relativistic $\left(k^{2}>m_{s}^{2}(R) a^{2}\right)$ at recent redshifts. Since $\gamma$ has a characteristic time and wavenumber dependence, future detailed observations may yield useful information on the validity of $f(R)$ gravity through this quantity, although current constraints have been obtained assuming that it is constant both in time and in wavenumber[12].

We have also numerically confirmed a shift in the power spectrum index for larger wavenumbers which exceed the scalaron mass during the matter-dominated epoch[13], while for smaller wavenumbers, fluctuations have the same amplitude as in the $\Lambda$ CDM model. Once more, the future de Sitter epoch stability condition bounds a possible increase in density fluctuations for cluster scales (compared with the $\Lambda$ CDM model) by $\sim 40 \%$ for $n \geq 2$. This enhancement in matter power spectrum can explain the observational data from SDSS and allows for some increase in the total sum of neutrino restmasses, as compared to the standard $\Lambda$ CDM model.

\section{Acknowledgments}

AS acknowledges RESCEU hospitality as a visiting professor. He was also partially supported by the grant RFBR 11-02-00643 and by the Scientific Programme "Astronomy" of the Russian Academy of Sciences. This work was supported in part by JSPS Research Fellowships for Young Scientists (HM), JSPS Grant-in-Aid for Scientific Research No. 19340054 (JY), Grant-in-Aid for Scientific Research on Innovative Areas No. 21111006 (JY), JSPS Core-to-Core program "International Research Network on Dark Energy", and Global COE 
Program "the Physical Sciences Frontier", MEXT, Japan.

[1] E. Komatsu et al., [arXiv:1001.4538].

[2] A. A. Starobinsky, Phys. Lett. 91B, 99 (1980).

[3] K. Sato, Mon. Not. Roy. Astron. Soc. 195, 467 (1981).

[4] A. H. Guth, Phys. Rev. D 23, 347 (1981).

[5] W. Hu and I. Sawicki, Phys. Rev. D 76, 064004 (2007) [arXiv:0705.1158].

[6] A. Appleby and R. Battye, Phys. Lett. B 654, 7 (2007) [arXiv:0705.3199].

[7] A. A. Starobinsky, JETP Lett. 86, 157 (2007) [arXiv:0706.2041].

[8] V. Müller, H.-J. Schmidt and A. A. Starobinsky, Phys. Lett. B 202, 198 (1988).

[9] M. Chevallier and D. Polarski, Int. J. Mod. Phys. D 10, 213 (2001) [gr-qc/0009008]; E. V. Linder, Phys. Rev. Lett. 90, 091301 (2003) [astro-ph/0208512].

[10] P. Zhang, Phys. Rev. D 73, 123504 (2006) [astro-ph/0511218].

[11] S. Tsujikawa, Phys. Rev. D 76, 023514 (2007) [arXiv:0705.1032].

[12] D. Rapetti, S. W. Allen, A. Mantz and H. Ebeling, MNRAS 406, 1796 (2010) [arXiv:0911.1787].

[13] H. Motohashi, A. A. Starobinsky and J. Yokoyama, Int. J. Mod. Phys. D 18, 1731 (2009) [arXiv:0905.0730].

[14] H. Motohashi, A. A. Starobinsky and J. Yokoyama, Prog. Theor. Phys. 123, 887 (2010) [arXiv:1002.1141].

[15] H. Motohashi, A. A. Starobinsky and J. Yokoyama, Prog. Theor. Phys. 124, 541 (2010) [arXiv:1005.1171].

[16] H. Motohashi, A. A. Starobinsky and J. Yokoyama, in preparation.

[17] B. A. Reid et al., Mon. Not. Roy. Astron. Soc. 404, 60 (2010) [arXiv:0907.1659].

[18] A. Shafieloo, V. Sahni and A. A. Starobinsky, Phys. Rev. D 80, 101301 (R) (2009) [arXiv:0903.5141]. 